\title{
Article \\ Clinical Outcomes in the Second versus First Pandemic Wave in Italy: Impact of Hospital Changes and Reorganization
}

\author{
Antonio Voza ${ }^{1,2, *}$, Antonio Desai ${ }^{1,2}{ }^{\mathbb{D}}$, Sabino Luzzi ${ }^{3,4}$, Alice Giotta Lucifero ${ }^{3}$, Elena Azzolini ${ }^{2,5}$, Maria Kogan ${ }^{1}$, \\ Giulia Goretti $^{1}$, Daniele Piovani ${ }^{2}$, Stefanos Bonovas ${ }^{2}$, Giovanni Angelotti ${ }^{6}$, Victor Savevski ${ }^{6}{ }^{D}$, \\ Alessio Aghemo ${ }^{2,7}$, Massimiliano Greco ${ }^{2,8}$, Elena Costantini ${ }^{8}$, Ana Lleo ${ }^{2,7}$ (D), Claudio Angelini ${ }^{9}$, \\ Mauro Giordano ${ }^{10}$, Salvatore Badalamenti ${ }^{9}$ and Maurizio Cecconi ${ }^{2,8}$
}

Citation: Voza, A.; Desai, A.; Luzzi, S.; Giotta Lucifero, A.; Azzolini, E.; Kogan, M.; Goretti, G.; Piovani, D.; Bonovas, S.; Angelotti, G.; et al. Clinical Outcomes in the Second versus First Pandemic Wave in Italy: Impact of Hospital Changes and Reorganization. Appl. Sci. 2021, 11, 9342. https://doi.org/10.3390/ app11199342

Academic Editor: Johann Michael Köhler

Received: 1 September 2021

Accepted: 4 October 2021

Published: 8 October 2021

Publisher's Note: MDPI stays neutral with regard to jurisdictional claims in published maps and institutional affiliations.

Copyright: (c) 2021 by the authors. Licensee MDPI, Basel, Switzerland. This article is an open access article distributed under the terms and conditions of the Creative Commons Attribution (CC BY) license (https:/ / creativecommons.org/licenses/by/ $4.0 /)$.
1 Emergency Department, IRCCS Humanitas Research Hospital, Via Manzoni 56, 20089 Milan, Italy; desaiantonio@gmail.com (A.D.); maria.kogan@humanitas.it (M.K.); giulia.goretti@humanitas.it (G.G.)

2 Department of Biomedical Sciences, Humanitas University, Via Rita Levi Montalcini 4, 20090 Milan, Italy; elena.azzolini@humanitas.it (E.A.); daniele.piovani@humanitas.it (D.P.); stefanos.bonovas@humanitas.it (S.B.); alessio.aghemo@hunimed.eu (A.A.); massimiliano.greco@humanitas.it (M.G.); ana.lleo@humanitas.it (A.L.); maurizio.cecconi@hunimed.eu (M.C.)

3 Neurosurgery Unit, Department of Clinical-Surgical, Diagnostic and Pediatric Sciences, University of Pavia, 27100 Pavia, Italy; sabino.luzzi@unipv.it (S.L.); alicelucifero@gmail.com (A.G.L.)

4 Neurosurgery Unit, Department of Surgical Sciences, Fondazione IRCCS Policlinico San Matteo, 27100 Pavia, Italy

5 Health Directorate, IRCCS Humanitas Research Hospital, Via Manzoni 56, 20089 Milan, Italy

6 Artificial Intelligence Center, IRCCS Humanitas Research Hospital, Via Manzoni 56, 20089 Milan, Italy; giovanni.angelotti@humanitas.it (G.A.); victor.savevski@humanitas.it (V.S.)

7 Department of Internal Medicine and Hepatology, IRCCS Humanitas Research Hospital, Via Manzoni 56, 20089 Milan, Italy

8 Department and Anaesthesia and Intensive Care, IRCCS Humanitas Research Hospital, Via Manzoni 56, 20089 Milan, Italy; elena.costantini@humanitas.it

9 Department of Nephrology, IRCCS Humanitas Research Hospital, via Manzoni 56, 20089 Milan, Italy; claudio.angelini@humanitas.it (C.A.); salvatore.badalamenti@humanitas.it (S.B.)

10 Department of Advanced Medical and Surgical Sciences, University of Campania "L. Vanvitelli", 81100 Naples, Italy; mauro.giordano.65@gmail.com

* Correspondence: antonio.voza@humanitas.it; Tel.: +39-028-224-8357 or +39-335-640-2791

Abstract: The region of Lombardy was the epicenter of the COVID-19 outbreak in Italy. Emergency Hospital 19 (EH19) was built in the Milan metropolitan area during the pandemic's second wave as a facility of Humanitas Clinical and Research Center (HCRC). The present study aimed to assess whether the implementation of EH19 was effective in improving the quality of care of COVID-19 patients during the second wave compared with the first one. The demographics, mortality rate, and in-hospital length of stay (LOS) of two groups of patients were compared: the study group involved patients admitted at HCRC and managed in EH19 during the second pandemic wave, while the control group included patients managed exclusively at HCRC throughout the first wave. The study and control group included 903 (56.7\%) and 690 (43.3\%) patients, respectively. The study group was six years older on average and had more pre-existing comorbidities. EH19 was associated with a decrease in the intensive care unit admission rate $(16.9 \%$ vs. $8.75 \%, p<0.001)$, and an equal decrease in invasive oxygen therapy $(3.8 \%$ vs. $0.23 \%, p<0.001)$. Crude mortality was similar but overlap propensity score weighting revealed a trend toward a potential small decrease. The adjusted difference in LOS was not significant. The implementation of an additional COVID-19 hospital facility was effective in improving the overall quality of care of COVID-19 patients during the first wave of the pandemic when compared with the second. Further studies are necessary to validate the suggested approach.

Keywords: COVID-19; emergency department; lean; management algorithm; SARS-CoV-2 


\section{Introduction}

The Coronavirus Disease 2019 (COVID-19) outbreak had a significant impact on the global health system in Italy and worldwide, as a pandemic of this scale had not been observed in the past 100 years. The Lombardy region, in northern Italy, was the epicenter of the pandemic on the Italian national territory and one of the most afflicted areas worldwide [1-5]. Herein, the irrepressible spread of the Severe Acute Respiratory Syndrome Coronavirus 2 (SARS-CoV-2) infection created the immediate necessity for logistic, organizational, and management adjustments within most hospitals, which were caught unprepared for an event without precedent in the national health system [6-14]. Several public and private hospitals responded to the increased demand by internal rearrangements of their routine daily activities. Some hospitals built extra capacity in preparation for a second wave. Our hospital built a new hospital facility on the hospital campus in 8 weeks intending to increase capacity and optimize infection containment in the hospital. To our knowledge, this is the first European COVID-19 hospital built in response to the pandemic.

Furthermore, the description of these rare adjustments at the hospital level still lacks in the literature, in terms of their effects on overall mortality and in-hospital length of stay (LOS). After the first wave of COVID-19, a hospital facility was strategically built in southern Milan by Humanitas Clinical and Research Center (ICH), a private hospital contracted to the National Health System and has a vast catchment area, to face the second and potential following waves. The new hospital facility, called Humanitas Emergency Hospital 19 (EH19), is in a different urban area from the ICH. Inaugurated on 27 July 2020, EH19 was equipped with 17 inpatient rooms, a dedicated emergency department, a radiology unit, an operating room, and an intensive care unit (ICU) consisting of 12 beds. Moreover, a high-dependency unit, in charge of the Emergency Department team, equipped with eight beds was also built. These eight beds had the potential to be used as ICU beds in case of necessity during a surge. The rationale of EH19 was to provide logistic support to the multidisciplinary team of specialists engaged in the treatment of COVID-19 patients and simultaneously facilitate an immunological research program for the cure of SARSCoV-2 infection. The key resident teams were the Emergency Medicine team and the ICU team. Other teams were available on a consultation basis and deployed by the nearby main hospital. These teams worked closely together to ensure patients received the best care and evaluate each decision on a case-by-case basis.

From an operational standpoint, EH19 was inspired by the evidence-based and worldwide accepted "Lean method", which was practically realized through a stepwise process now known by the term "Lean-ing" [7,15,16].

The present study aimed to assess whether the implementation of an additional COVID-19 facility in a tertiary Milan hospital, such as EH19, and its use of Lean-ing were effective in improving the overall quality of care of patients with SARS-CoV-2 infection in the second wave of COVID-19 when compared with the first one.

\section{Methods}

\subsection{Study Design}

The present single-center retrospective cohort study was conducted at the Humanitas Clinical and Research Center, Milan, Lombardy Region, northern Italy, and obtained the approval of the local ethical committee, in agreement with the principles of the Helsinki declaration [17].

All the patients admitted to the Emergency Department (ED) from 1 March 2020, to 1 February 2021, were derived from an electronic database. Only patients who underwent testing with a nasopharyngeal swab at admission due to a suspected diagnosis of COVID19 were selected. Patients were divided into two groups based on the date of the admission and intra- and inter-hospital management protocol, as depicted below.

The first group included patients who were admitted at HCRC between 1 March 2020, and 31 May 2020; at the time the EH19 hospital facility was still unavailable. Patients who presented with COVID-19 symptoms at triage level were transferred to a grey area in 
which pulse oximetry and nasopharyngeal swab were performed. The peripheral oxygen saturation (SpO2) guided patient allocation to a high-, medium-, or low-intensity area, whereas, in the case of positivity for SARS-CoV-2 infection ascertained with polymerase chain reaction, the patients were assigned to a COVID-19 ICU or ward in the same hospital. The second group was comprised of the patients admitted at HCRC from 1 October 2020, to 1 February 2021. It was allocated based on the same model, but patients were transferred to and managed in EH19 if suspected of SARS-CoV-2 infection. From a temporal point of view, the groups were samples of the first and second waves of COVID-19, in northern Italy, respectively. Diagrams 1 and 2 summarize the management algorithms used in groups 1 and 2, respectively (Figures 1 and 2). In both groups, the age, gender, signs and symptoms at hospital admission, comorbidities, blood routine, infection-related biomarkers, blood biochemistry, and coagulation profile data were collected. To assess the overall quality of care of COVID-19 patients between the first and second wave, data were compared regarding the mortality rate and in-hospital LOS.

\section{OPERATIONAL ALGORITHM DURING FIRST COVID-19 WAVE}

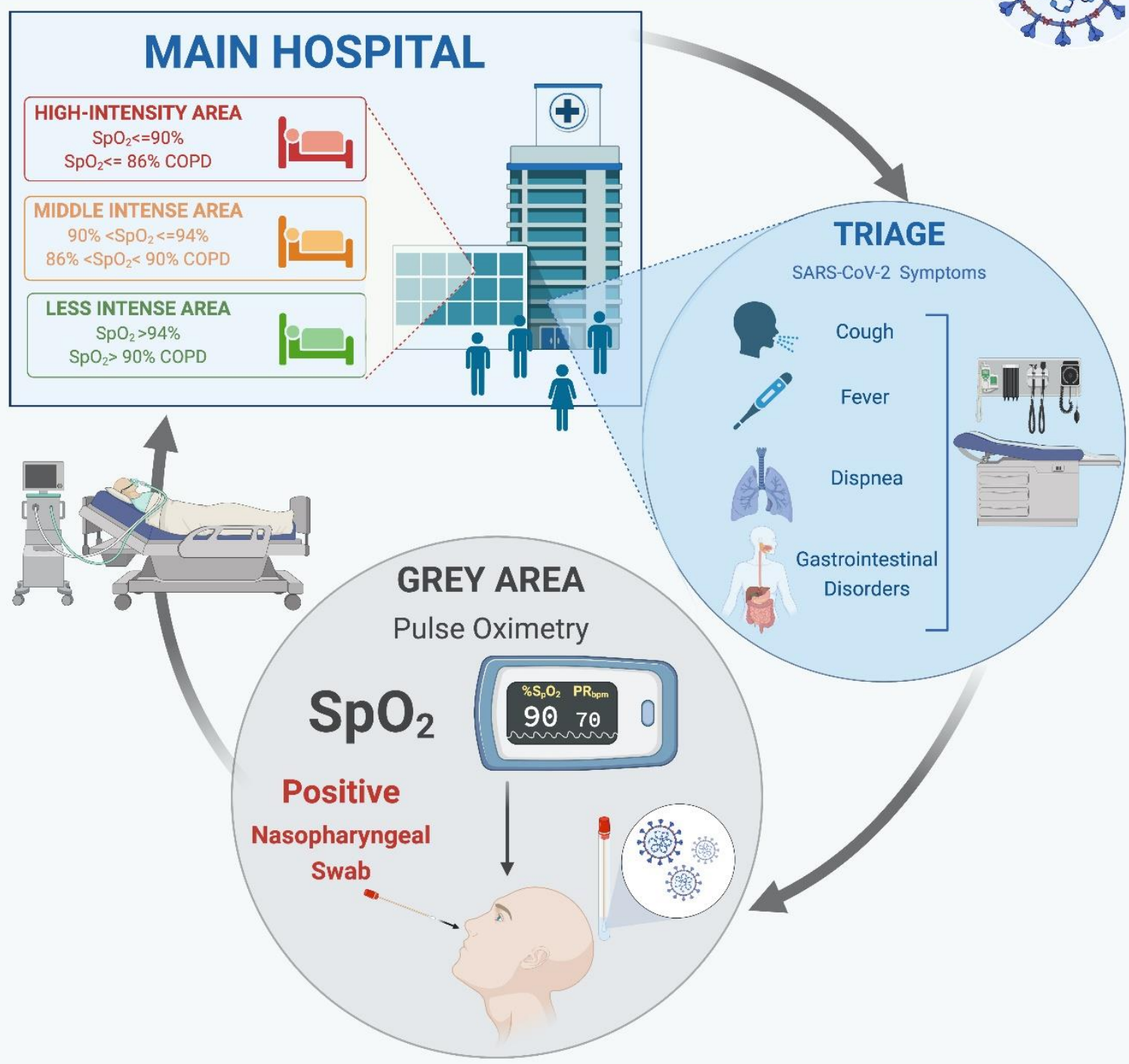

Figure 1. Operational algorithm used during the first wave of COVID-19. 


\section{OPERATIONAL ALGORITHM DURING SECOND COVID-19 WAVE}

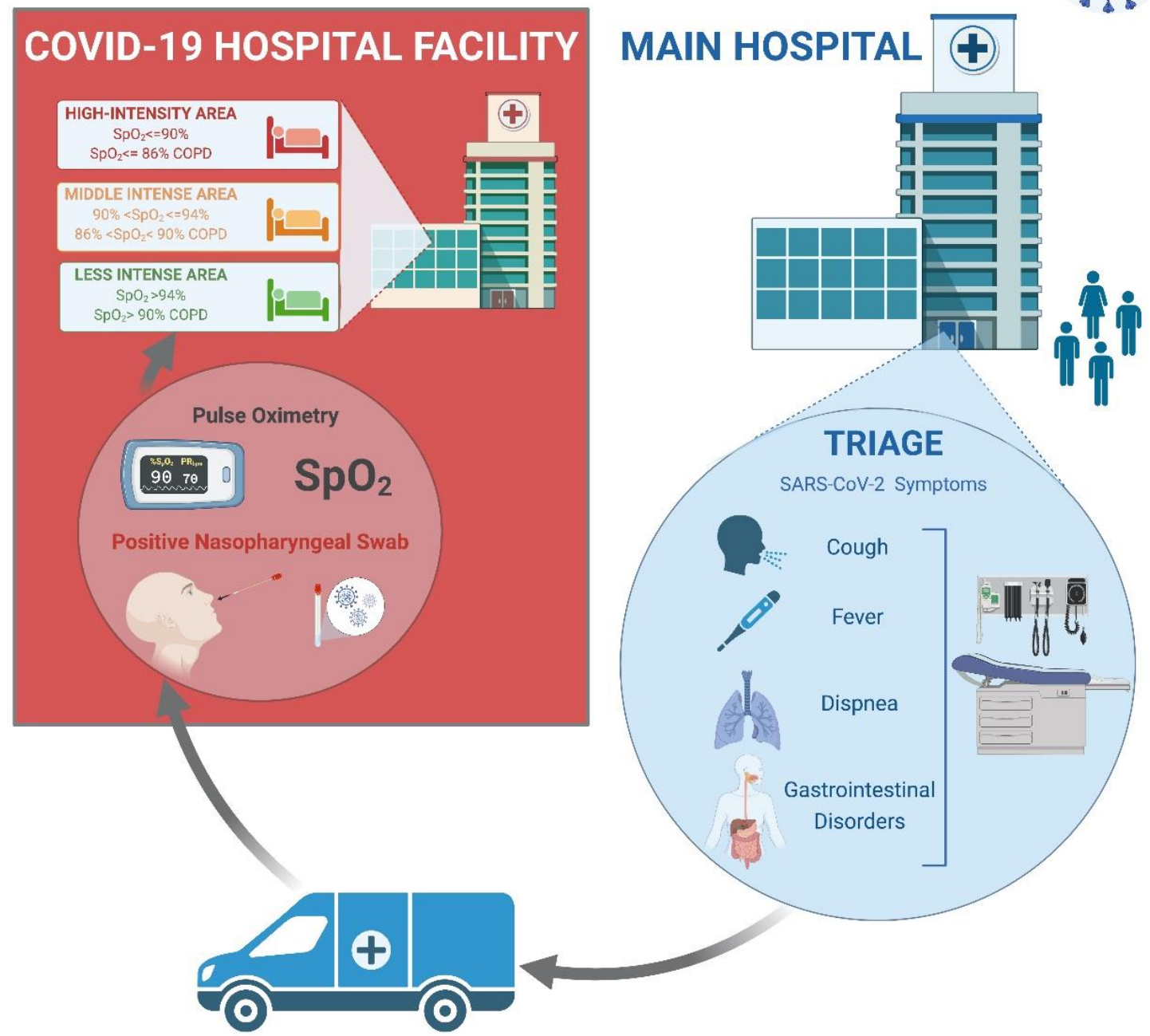

Figure 2. Operational algorithm used during the second wave of COVID-19.

\subsection{Statistical Analysis}

Descriptive statistics included means with standard deviations (SD) and medians with interquartile ranges (IQR) for continuous variables and counts (percentages) for categorical variables. For the univariable comparison of demographic, clinical, and laboratory variables among the two groups (first vs. second wave), a non-parametric Wilcoxon rank-sum test was used for numeric variables, and a $\chi^{2}$ test was used for categorical variables. A propensity score belonging to the first or second wave of COVID-19 was estimated from a multivariable logistic regression model including patient age, gender, presence of hypertension, cardiovascular diseases, diabetes, malignancies, neurological diseases, respiratory diseases, and chronic kidney diseases. Considering that age and underlying comorbidities are strongly associated with mortality and LOS, and that they were unbalanced between the first and second waves of COVID-19 patients, an overlap propensity score weighting was performed to address potential confounding factors $[18,19]$.

The overlap propensity score-weighted logistic regression model investigated the in-hospital mortality and LOS in the patients of groups 1 and 2. As the distribution of the LOS was severely skewed, including the residuals deriving from the multivariable regression, we logged this outcome variable to achieve normalization. Stata 15.0 software (Stata Corp., College Station, TX, USA) and R (https:/ / www.r-project.org accessed on 
1 February 2021) were used to analyze the data. $p$-values $<0.05$ were considered statistically significant. All tests were two-sided.

\section{Results}

The data set consisted of 1593 COVID-19 patients with complete follow-ups who were admitted to the Humanitas Research Hospital, Italy.

The mean (SD) age was 69.0 (14.6) years (median 71, IQR: 59-80), and 64.2\% were males. A substantial proportion of patients was affected by at least one comorbidity at baseline $(75.9 \%)$, including, in particular, hypertension (50.7\%), cardiovascular diseases $(26.6 \%)$, diabetes $(19.4 \%)$, malignancy $(19.4 \%)$, neurological diseases $(17.3 \%)$, respiratory diseases $(14.4 \%)$ and chronic kidney diseases $(9.4 \%)$. Among these hospitalized patients, $10.1 \%$ received continuous positive airway pressure therapy (CPAP), $12.3 \%$ were admitted to the intensive care unit, and $24.0 \%$ died. The mean (SD) LOS was 16.2 (15.6) days (median 11, IQR: 7-19). The mean LOS during the first wave were 12.3 days and 18.4 days in the emergency ward and ICU, respectively. On the contrary, during the second wave, the mean LOS in the emergency ward was 14.1 days, and 11.6 in the ICU.

\subsection{Patients' Characteristics and Interventions Received in the First and Second Wave}

Of the 1593 included in the analyses, 690 (43.3\%) were admitted during the first wave and 903 (56.7\%) during the second wave. We reported baseline characteristics at the admission of the two cohorts of patients including demographic, clinical, and laboratory findings in Table 1. Patients in the second wave were a median of six years older and had significantly more pre-existing comorbidities than those hospitalized in the first wave. Additionally, the proportion of women was higher in the second wave. Laboratory characteristics confirmed the substantial difference between these two cohorts of patients with significantly different values in lymphocytes, eosinophils, hemoglobin, interleukin-6, serum ferritin, c-reactive protein, gamma-glutamyl transpeptidase, creatine kinase, triglycerides, international normalized ratio, and fibrinogen, Table 1 . The new internal organization resulted in a lower proportion of patients admitted to the ICU in the second wave (first vs. second wave, $16.9 \%$, and $8.75 \%, p<0.001$ ) mostly substituted by a sub-intensive type of care. The proportion of patients receiving invasive oxygen therapy $(3.8 \%$ vs. $0.23 \%, p<0.001)$ was significantly lower in the second wave, while the proportion of those receiving other types of oxygen support was significantly higher ( $54.3 \%$ vs. $63.1 \%, p=0.001)$. The use of CPAP was not significantly different between the two waves $(11.9 \%$ vs. $9.1 \%, p=0.189)$.

Table 1. Demographic, clinical, and laboratory characteristics of hospitalized COVID-19 patients $(n=1593)$.

\begin{tabular}{|c|c|c|c|}
\hline & First Wave $(n=690)$ & Second Wave $(n=903)$ & \multirow{2}{*}{$p$-Value } \\
\hline & Mean \pm SD; Median (IQR) or $\mathbf{n}(\%)$ & Mean \pm SD; Median (IQR) or $n(\%)$ & \\
\hline \multicolumn{4}{|c|}{ Demographic Characteristics } \\
\hline Age (years) & $\begin{array}{c}66.21 \pm 14.44 ; 67.00(56.00-77.00) \\
(\mathrm{n}=690)\end{array}$ & $\begin{array}{c}71.17 \pm 14.30 ; 73.00(61.00-82.00) \\
(\mathrm{n}=903)\end{array}$ & $<0.01$ \\
\hline$\geq 65$ & $\begin{array}{l}296(42.9) \\
(n=690)\end{array}$ & $\begin{array}{l}272(30.1) \\
(\mathrm{n}=903)\end{array}$ & \\
\hline$<65$ & $\begin{array}{l}394(57.1) \\
(\mathrm{n}=690)\end{array}$ & $\begin{array}{l}631(69.9) \\
(\mathrm{n}=903)\end{array}$ & \\
\hline Gender & & & 0.006 \\
\hline Female & $\begin{array}{l}221(32.0) \\
(\mathrm{n}=690)\end{array}$ & $\begin{array}{l}350(38.8) \\
(\mathrm{n}=903)\end{array}$ & \\
\hline Male & $\begin{array}{l}469(68.0) \\
(n=690)\end{array}$ & $\begin{array}{l}553(61.2) \\
(\mathrm{n}=903)\end{array}$ & \\
\hline
\end{tabular}


Table 1. Cont.

\begin{tabular}{|c|c|c|c|}
\hline & First Wave $(n=690)$ & Second Wave $(\mathrm{n}=903)$ & \\
\hline & Mean \pm SD; Median (IQR) or $\mathbf{n}(\%)$ & Mean \pm SD; Median (IQR) or $\mathbf{n}(\%)$ & $p$-value \\
\hline \multicolumn{4}{|l|}{ Clinical Characteristics } \\
\hline \multicolumn{4}{|l|}{$\begin{array}{c}\text { Signs and Symptoms at } \\
\text { Admission }\end{array}$} \\
\hline $\begin{array}{l}\text { Systolic blood pressure } \\
(\mathrm{mmHg})\end{array}$ & $\begin{array}{c}134.55 \pm 156.80 ; 125.79(116.88-138.17) \\
(\mathrm{n}=612)\end{array}$ & $\begin{array}{c}177.25 \pm 1414.32 ; 127.00(117.50-137.25) \\
(\mathrm{n}=821)\end{array}$ & 0.415 \\
\hline Pulse (beats per minute) & $\begin{array}{c}84.35 \pm 11.86 ; 83.79(76.00-91.73) \\
(n=612)\end{array}$ & $\begin{array}{c}84.35 \pm 27.40 ; 81.67(74.20-91.05) \\
(n=823)\end{array}$ & 0.012 \\
\hline $\begin{array}{l}\text { Respiratory rate (breaths per } \\
\text { minute) }\end{array}$ & $\begin{array}{c}19.15 \pm 3.22 ; 18.33(17.50-20.00) \\
(\mathrm{n}=586)\end{array}$ & $\begin{array}{c}18.87 \pm 3.46 ; 18.00(17.40-19.50) \\
(\mathrm{n}=769)\end{array}$ & 0.076 \\
\hline Pulse oximetry (\%) & $\begin{array}{c}93.51 \pm 11.66 ; 95.33(92.00-97.00) \\
(n=550)\end{array}$ & $\begin{array}{c}92.33 \pm 6.48 ; 94.00(90.00-96.57) \\
(\mathrm{n}=765)\end{array}$ & $<0.01$ \\
\hline Glasgow coma scale & $\begin{array}{c}14.75 \pm 1.38 ; 15.00(15.00-15.00) \\
(\mathrm{n}=615)\end{array}$ & $\begin{array}{c}14.83 \pm 0.86 ; 15.00(15.00-15.00) \\
(\mathrm{n}=820)\end{array}$ & 0.140 \\
\hline \multicolumn{4}{|l|}{ Comorbid Conditions } \\
\hline Any comorbidity & $\begin{array}{l}482(70.6) \\
(\mathrm{n}=683)\end{array}$ & $\begin{array}{l}722(80.0) \\
(\mathrm{n}=903)\end{array}$ & $<0.01$ \\
\hline Hypertension & $\begin{array}{l}321(47.1) \\
(\mathrm{n}=682)\end{array}$ & $\begin{array}{l}482(53.5) \\
(\mathrm{n}=901)\end{array}$ & 0.013 \\
\hline Cardiovascular diseases & $\begin{array}{l}168(24.6) \\
(\mathrm{n}=683)\end{array}$ & $\begin{array}{l}252(28.1) \\
(\mathrm{n}=898)\end{array}$ & 0.137 \\
\hline Diabetes & $\begin{array}{l}147(21.5) \\
(\mathrm{n}=683)\end{array}$ & $\begin{array}{l}160(17.8) \\
(\mathrm{n}=899)\end{array}$ & 0.100 \\
\hline Malignancy & $\begin{array}{l}113(16.5) \\
(\mathrm{n}=683)\end{array}$ & $\begin{array}{l}194(21.6) \\
(\mathrm{n}=899)\end{array}$ & 0.015 \\
\hline Neurological diseases & $\begin{array}{l}97(14.3) \\
(\mathrm{n}=679)\end{array}$ & $\begin{array}{l}176(19.6) \\
(\mathrm{n}=897)\end{array}$ & 0.007 \\
\hline Respiratory diseases & $\begin{array}{l}92(13.5) \\
(\mathrm{n}=683)\end{array}$ & $\begin{array}{l}136(15.1) \\
(\mathrm{n}=900)\end{array}$ & 0.396 \\
\hline Chronic kidney diseases & $\begin{array}{c}54(7.9) \\
(\mathrm{n}=681)\end{array}$ & $\begin{array}{l}94(10.4) \\
(\mathrm{n}=900)\end{array}$ & 0.107 \\
\hline \multicolumn{4}{|l|}{ Laboratory Characteristics } \\
\hline \multicolumn{4}{|l|}{ Blood Routine } \\
\hline $\begin{array}{l}\text { White blood cells }\left(\times 10^{9} / \mathrm{L} \text {; }\right. \\
\text { normal range } 4.0-10.0)\end{array}$ & $\begin{array}{c}8.45 \pm 7.33 ; 6.89(5.22-10.08) \\
(n=638)\end{array}$ & $\begin{array}{c}8.83 \pm 12.85 ; 7.40(5.39-10.12) \\
(\mathrm{n}=866)\end{array}$ & 0.998 \\
\hline $\begin{array}{c}\text { Eosinophils }\left(\times 10^{9} / \mathrm{L} ; \text { normal }\right. \\
\text { range } 0.0-0.5)\end{array}$ & $\begin{array}{c}0.03 \pm 0.09 ; 0.00(0.00-0.00) \\
(\mathrm{n}=650)\end{array}$ & $\begin{array}{c}0.02 \pm 0.06 ; 0.00(0.00-0.00) \\
(\mathrm{n}=881)\end{array}$ & 0.027 \\
\hline $\begin{array}{c}\text { Monocytes }\left(\times 10^{9} / \mathrm{L} ; \text { normal }\right. \\
\text { range } 0.2-0.8)\end{array}$ & $\begin{array}{l}0.63 \pm 0.65 ; 0.50(0.40-0.80) \\
(\mathrm{n}=650)\end{array}$ & $\begin{array}{c}0.70 \pm 2.17 ; 0.50(0.40-0.80) \\
(\mathrm{n}=881)\end{array}$ & 0.059 \\
\hline $\begin{array}{l}\text { Basophils }\left(\times 10^{9} / \mathrm{L} \text {; normal }\right. \\
\text { range } 0.0-0.1)\end{array}$ & $\begin{array}{c}0.01 \pm 0.04 ; 0.00(0.00-0.00) \\
(\mathrm{n}=650)\end{array}$ & $\begin{array}{c}0.02 \pm 0.04 ; 0.00(0.00-0.00) \\
(\mathrm{n}=881)\end{array}$ & 0.330 \\
\hline $\begin{array}{l}\text { Lymphocytes }\left(\times 10^{9} / \mathrm{L} \text {; }\right. \\
\text { normal range } 1.0-4.0)\end{array}$ & $\begin{array}{c}1.31 \pm 5.31 ; 0.90(0.60-1.30) \\
(\mathrm{n}=650)\end{array}$ & $\begin{array}{l}1.40 \pm 11.12 ; 0.90(0.60-1.20) \\
(\mathrm{n}=881)\end{array}$ & $<0.01$ \\
\hline $\begin{array}{l}\text { Platelets }\left(\times 10^{9} / \mathrm{L} \text {; normal }\right. \\
\quad \text { range } 150-400)\end{array}$ & $\begin{array}{c}228.09 \pm 105.68 ; 212.00(158.00-275.50) \\
(\mathrm{n}=651)\end{array}$ & $\begin{array}{c}228.83 \pm 106.86 ; 211.00(161.00-277.00) \\
(\mathrm{n}=881)\end{array}$ & 0.338 \\
\hline $\begin{array}{l}\text { Hemoglobin }(\mathrm{g} / \mathrm{dL} ; \text { normal } \\
\text { range } 13.0-16.0)\end{array}$ & $\begin{array}{c}13.44 \pm 2.04 ; 13.70(12.30-14.80) \\
(\mathrm{n}=651)\end{array}$ & $\begin{array}{c}13.22 \pm 2.13 ; 13.40(12.00-14.60) \\
(\mathrm{n}=881)\end{array}$ & 0.001 \\
\hline
\end{tabular}


Table 1. Cont.

\begin{tabular}{|c|c|c|c|}
\hline & First Wave $(n=690)$ & Second Wave $(n=903)$ & \multirow{2}{*}{$p$-Value } \\
\hline & Mean \pm SD; Median (IQR) or $\mathbf{n}(\%)$ & Mean \pm SD; Median (IQR) or $\mathbf{n}(\%)$ & \\
\hline \multicolumn{4}{|l|}{$\begin{array}{l}\text { Infection-Related } \\
\text { Biomarkers }\end{array}$} \\
\hline $\begin{array}{l}\text { Procalcitonin }(\mathrm{ng} / \mathrm{mL} ; \text { normal } \\
\text { range } 0.05-0.5)\end{array}$ & $\begin{array}{c}0.89 \pm 3.23 ; 0.21(0.10-0.50) \\
(\mathrm{n}=550)\end{array}$ & $\begin{array}{c}2.33 \pm 19.30 ; 0.20(0.10-0.54) \\
(\mathrm{n}=695)\end{array}$ & 0.312 \\
\hline $\begin{array}{c}\text { Interleukin-6 }(\mathrm{pg} / \mathrm{mL} ; \text { normal } \\
\text { range }<6.4)\end{array}$ & $\begin{array}{c}86.49 \pm 148.17 ; 45.00(19.00-93.00) \\
(n=453)\end{array}$ & $\begin{array}{c}72.37 \pm 152.93 ; 40.00(19.00-76.25) \\
(\mathrm{n}=772)\end{array}$ & $<0.01$ \\
\hline $\begin{array}{l}\text { Serum ferritin }(\mathrm{ng} / \mathrm{mL} ; \\
\text { normal range } 23.9-336.2)\end{array}$ & $\begin{array}{c}740.96 \pm 799.50 ; 500.40(209.00-962.97) \\
(\mathrm{n}=570)\end{array}$ & $\begin{array}{c}704.97 \pm 905.69 ; 456.35(215.55-884.62) \\
(\mathrm{n}=800)\end{array}$ & 0.002 \\
\hline $\begin{array}{l}\text { C-reactive protein }(\mathrm{mg} / \mathrm{dL} ; \\
\text { normal range }<1.0)\end{array}$ & $\begin{array}{c}10.23 \pm 8.98 ; 8.17(2.80-15.01) \\
(\mathrm{n}=615)\end{array}$ & $\begin{array}{c}9.49 \pm 8.17 ; 8.34(2.64-13.34) \\
(\mathrm{n}=848)\end{array}$ & 0.020 \\
\hline \multicolumn{4}{|l|}{ Blood Biochemistry } \\
\hline $\begin{array}{l}\text { Alanine aminotransferase } \\
(\mathrm{U} / \mathrm{L} \text {; normal range < 51) }\end{array}$ & $\begin{array}{c}38.88 \pm 45.86 ; 27.00(18.00-44.00) \\
(n=624)\end{array}$ & $\begin{array}{c}40.69 \pm 60.82 ; 26.00(17.00-45.00) \\
(n=846)\end{array}$ & 0.046 \\
\hline $\begin{array}{l}\text { Aspartate aminotransferase } \\
(\mathrm{U} / \mathrm{L} \text {; normal range < 51) }\end{array}$ & $\begin{array}{c}48.15 \pm 49.07 ; 36.00(25.00-54.00) \\
(\mathrm{n}=567)\end{array}$ & $\begin{array}{c}48.52 \pm 56.80 ; 35.00(25.00-51.00) \\
(n=764)\end{array}$ & 0.410 \\
\hline $\begin{array}{c}\text { Gamma-glutamyl } \\
\text { transpeptidase }(\mathrm{U} / \mathrm{L} \text {; normal } \\
\text { range }<55)\end{array}$ & $\begin{array}{c}68.57 \pm 78.67 ; 43.00(22.00-81.25) \\
\mathrm{n}=576)\end{array}$ & $\begin{array}{c}80.35 \pm 133.35 ; 45.00(25.00-85.00) \\
(n=679)\end{array}$ & $<0.01$ \\
\hline $\begin{array}{l}\text { Alkaline phosphatase }(\mathrm{U} / \mathrm{L} ; \\
\text { normal range } 40-150)\end{array}$ & $\begin{array}{c}101.29 \pm 120.89 ; 80.00(63.00-110.00) \\
(\mathrm{n}=562)\end{array}$ & $\begin{array}{c}103.99 \pm 71.22 ; 85.00(67.00-115.00) \\
(\mathrm{n}=773)\end{array}$ & 0.316 \\
\hline $\begin{array}{l}\text { Total bilirubin }(\mathrm{mg} / \mathrm{dL} ; \\
\text { normal range } 0.3-1.2)\end{array}$ & $\begin{array}{c}0.79 \pm 0.75 ; 0.70(0.50-0.90) \\
(\mathrm{n}=571)\end{array}$ & $\begin{array}{c}0.86 \pm 1.08 ; 0.70(0.50-0.90) \\
(\mathrm{n}=762)\end{array}$ & 0.283 \\
\hline $\begin{array}{l}\text { Direct bilirubin }(\mathrm{mg} / \mathrm{dL} ; \\
\text { normal range }<0.3)\end{array}$ & $\begin{array}{c}0.27 \pm 0.46 ; 0.20(0.10-0.30) \\
(\mathrm{n}=507)\end{array}$ & $\begin{array}{c}0.30 \pm 0.74 ; 0.20(0.10-0.30) \\
(\mathrm{n}=660)\end{array}$ & 0.103 \\
\hline $\begin{array}{l}\text { Indirect bilirubin }(\mathrm{mg} / \mathrm{dL} \\
\text { normal range } 0.05-1.10)\end{array}$ & $\begin{array}{c}0.63 \pm 0.39 ; 0.50(0.40-0.70) \\
(\mathrm{n}=546)\end{array}$ & $\begin{array}{c}0.62 \pm 0.62 ; 0.50(0.40-0.70) \\
(\mathrm{n}=652)\end{array}$ & 0.431 \\
\hline $\begin{array}{l}\text { Serum creatinine }(\mathrm{mg} / \mathrm{dL} \\
\text { normal range } 0.67-1.17)\end{array}$ & $\begin{array}{c}1.19 \pm 1.05 ; 0.93(0.75-1.21) \\
(n=627)\end{array}$ & $\begin{array}{c}1.25 \pm 1.10 ; 0.96(0.78-1.30) \\
(n=853)\end{array}$ & 0.866 \\
\hline $\begin{array}{l}\text { Creatine kinase }(\mathrm{U} / \mathrm{L} \text {; normal } \\
\text { range }<172)\end{array}$ & $\begin{array}{c}222.78 \pm 454.01 ; 101.00(56.00-203.00) \\
(\mathrm{n}=623)\end{array}$ & $\begin{array}{c}179.11 \pm 313.68 ; 87.00(49.00-161.75) \\
(\mathrm{n}=846)\end{array}$ & $<0.01$ \\
\hline $\begin{array}{l}\text { Lactate dehydrogenase }(\mathrm{U} / \mathrm{L} ; \\
\text { normal range }<248)\end{array}$ & $\begin{array}{c}363.22 \pm 205.89 ; 314.50(240.00-416.25) \\
(\mathrm{n}=558)\end{array}$ & $\begin{array}{c}358.71 \pm 256.98 ; 312.00(243.00-416.50) \\
(\mathrm{n}=756)\end{array}$ & 0.284 \\
\hline $\begin{array}{l}\text { Triglycerides }(\mathrm{mg} / \mathrm{dL} \text {; normal } \\
\text { range } 10-150)\end{array}$ & $\begin{array}{c}139.10 \pm 88.36 ; 120.00(92.00-157.00) \\
(\mathrm{n}=540)\end{array}$ & $\begin{array}{c}137.10 \pm 80.02 ; 120.00(94.00-162.00) \\
(\mathrm{n}=773)\end{array}$ & 0.009 \\
\hline \multicolumn{4}{|l|}{$\begin{array}{l}\text { Coagulation Function and } \\
\text { Other Biomarkers }\end{array}$} \\
\hline $\begin{array}{l}\text { International Normalized } \\
\text { Ratio (normal range 0.9-1.18) }\end{array}$ & $\begin{array}{c}1.24 \pm 0.65 ; 1.11(1.05-1.22) \\
(\mathrm{n}=612)\end{array}$ & $\begin{array}{c}1.16 \pm 0.49 ; 1.08(1.02-1.16) \\
(\mathrm{n}=821)\end{array}$ & $<0.01$ \\
\hline $\begin{array}{l}\text { Fibrinogen }(\mathrm{mg} / \mathrm{dL} ; \text { normal } \\
\text { range } 160-400)\end{array}$ & $\begin{array}{c}561.69 \pm 183.65 ; 542.00(436.00-665.00) \\
(\mathrm{n}=577)\end{array}$ & $\begin{array}{c}597.48 \pm 196.11 ; 586.00(451.00-718.75) \\
(\mathrm{n}=782)\end{array}$ & 0.024 \\
\hline $\begin{array}{l}\text { D-dimer }(\mu \mathrm{g} / \mathrm{mL} ; \text { normal } \\
\text { range } 0.2-0.35)\end{array}$ & $\begin{array}{c}2.02 \pm 5.92 ; 0.54(0.33-1.00) \\
(\mathrm{n}=502)\end{array}$ & $\begin{array}{c}1.86 \pm 5.69 ; 0.46(0.30-0.98) \\
(\mathrm{n}=646)\end{array}$ & 0.455 \\
\hline $\begin{array}{l}\text { Troponin-I (ng/L; normal } \\
\text { range } 1-19.8)\end{array}$ & $\begin{array}{c}129.92 \pm 905.77 ; 10.20(4.30-28.00) \\
(\mathrm{n}=558)\end{array}$ & $\begin{array}{c}107.68 \pm 721.67 ; 11.80(6.60-28.20) \\
(\mathrm{n}=829)\end{array}$ & 0.307 \\
\hline
\end{tabular}




\subsection{Unadjusted and Overlap Propensity Scores Weighted Patients' Outcomes}

The crude mortality was $22.6 \%$ in the first and $25.1 \%$ in the second wave, with no statistically significant difference (second vs. the first wave, crude RR $1.11 ; 95 \%$ CI, $0.93-1.32 ; p=0.24$ ). After overlap propensity score weighting, the adjusted mortality was $24.9 \%$ in patients admitted during the first wave and $21.4 \%$ in those admitted during the second wave (second vs. the first wave, adjusted RR 0.86; 95\% CI, 0.73-1.02; $p=0.082$ ), showing suggestive evidence of a potential small decrease in mortality, Table 2.

Table 2. Overlap propensity score-weighted characteristics and in-hospital death among patients hospitalized for COVID-19 during the first or the second epidemic wave.

\begin{tabular}{ccc}
\hline & First Wave $^{\text {a }}$ & Second Wave $^{\text {a }}$ \\
\hline Age (years) & 68.2 & 68.2 \\
\hline Male (\%) & 65.1 & 65.1 \\
\hline Hypertension (\%) & 49.5 & 49.5 \\
\hline Cardiovascular diseases (\%) & 25.8 \\
\hline Diabetes (\%) & 20.1 & 20.1 \\
\hline Malignancy (\%) & 18.8 & 18.8 \\
\hline Neurological diseases (\%) & 16.6 \\
\hline Respiratory diseases (\%) & 16.6 & 13.9 \\
\hline Chronic kidney diseases (\%) & 8.5 \\
\hline In-hospital death & 13.9 & 21.4 \\
\hline RR (95\% CI) & 8.5 & \\
\hline OR (95\% CI) & 24.9 & \\
\hline
\end{tabular}

$\overline{{ }^{a}}$ Reported is either the overlap propensity score-weighted mean or proportion for each group.OR: Odds Ratio, RR: Relative Risk.

The unadjusted median length of stay was 10 days (IQR: 7-17) during the first wave and 12 days (IQR: 7-22) during the second wave, with a statistically significantly longer stay in the second wave (Wilcoxon rank-sum test; $p=0.023$ ). After overlap propensity score weighting, the adjusted difference in length of stay between the two waves was not statistically significant $(p=0.28)$.

\section{Discussion}

The present study describes the organizational aspects of EH19 as a way to better manage the COVID-19 pandemic. According to our experience, EH19 was very effective in facing the first and second waves as evidenced by a decrease in mortality and LOS, ultimately improving the overall quality of care. Based on data about global COVID-19 cases by John Hopkins CSSE updated at the end of the first wave, Italy was the third most-affected country of the world, only preceded by the US and Spain, accounting for 159,516 confirmed cases and 20,465 deaths [20]. Overall, the Lombardy region was the epicenter of the Italian pandemic, with $37.4 \%$ of total cases registered in the national territory and more than $50 \%$ of the total number of deaths (http://www.salute.gov.it accessed on 1 March 2021) [21-23].

The large number of cases in northern Italy overwhelmed the health care system and created a demand for an imminent change on the containment of the infection. After the implementation of EH19 and the Lean operational algorithm, the adjusted mortality rate was reduced, despite a seven-year increase in median patients' age and higher comorbidity rate in the second wave. Meanwhile, on the contrary, the need for HFO2 support increased. Moreover, the average in-hospital LOS was not prolonged in the second wave, resulting in a substantial advantage in terms of cost-effectiveness. These data may be explained by the overall improvement in the quality of care. 
As reported by several authors, the implementation of an additional hospital facility has a primary role in centralizing the professional figures specifically trained in the cure of COVID-19 patients [24-26]. COVID-19-dedicated hospitals have been proven to be effective in flattening the infection curve and slowing the spread of the virus worldwide- these being the major strengths of these facilities [24-27]. It should be mentioned that the ICU admission rate decreased in group $2(16.9 \%$ vs. $8.75 \%, p<0.001)$.

This could also be explained by considering that, in our newly born facility, besides the ordinary ward beds and the ICU beds, we had eight high-dependency unit beds at our disposal that could be easily converted to ICU or ordinary ward beds according to our needs with maximal flexibility.

The main sequelae of the COVID-19 spread included the alarming collapse of the clinic caseload and the consequent delays of elective medical and surgical activity. Roifman and colleagues recently stressed these concerns regarding the significant reduction in optimal cardiovascular health care delivery in Canada, basically consisting of a substantial deferral of elective cardiovascular procedures [28]. The same alarm has been reported for cardiac surgery [29], and as many apprehensions do exist for specialties other than the cardiovascular field, these reports highlight a negative trend in health care delivery, even in critical care [30-34]. A further advantage of the implementation of a satellite hospital dedicated to COVID-19 patients is that it aids in the decongestion of the main hospital, allowing the theoretical elective caseload to remain unaffected. EH19 also facilitated an environment where an in-depth anamnestic investigation of the patients' medical history, with a focus on comorbidities, was possible. This could explain the higher comorbidity rate among patients in the second wave when compared to the first. In other words, it avoids delaying the care of time-dependent pathologies and chronic diseases. Furthermore, due to ICH Cancer Center, patients with malignancies, who during the first wave of COVID-19 had been shielding inside their homes, have recently been accessing hospital care at an increasing rate, which allows for the continuity of treatment. This can partially explain the above-mentioned phenomenon.

Ferrara rightly emphasized the urgent need for a major reorganization of health care systems, in Italy and in other parts of the world, to allow the management of already anticipated waves as well as the continuity of the vaccination campaign from a medical and social point of view [35]. In line with this aim, the tactical build of COVID-19 facilities such as EH19 perfectly fits the aforementioned necessities. Additionally, strategic operational algorithms are mandatory, today and in the future, to optimize and further improve the quality of care of both COVID-19 and non-COVID-19 patients. The Lean model was adopted by our hospital, mainly because of its recognized high reliability, feasibility, and effectiveness [7,15,16,36-39]. It was reported as "an integrated socio-technical system whose main objective is to eliminate waste by concurrently reducing or minimizing supplier, customer, and internal variability" [40]. The motivating principles of this operative model make it an optimal choice for the management of the COVID-19 outbreak. It allowed for the successful screening of a large number of patients and for their proper allocation on Emergency Department arrival. There are clear limitations of this study due to its retrospective nature and the relatively small sample size. All of these limitations are common to other studies and are also caused by the exceptional situation generated by the SARS-CoV-2 pandemic.

\section{Limitations}

The present study has some limitations mainly due to its retrospective nature, the relatively limited number of patients managed, and the lack of randomization. Lastly, further limitations are the advances in treatment guidelines, which occurred in the second wave. The strengthening of target therapies and interventional strategies against COVID-19 could influence the LOS, ICU admission, and overall mortality during the second wave compared with the first one. 


\section{Conclusions}

Limited to the experience of a tertiary hospital in northern Italy, the implementation of an additional COVID-19 hospital facility and a strategic operative protocol were effective in the creation of logistic and organizational support designed to improve the overall quality of care of COVID-19 patients during the first wave of the pandemic when compared with the second.

The Lean model resulted in health care optimization through an improvement of the operative flows and the prevention of overcrowding in the ED.

Further studies are necessary to validate the presented approach relative to the containment of the SARS-CoV-2 infection, spreading, flattening of the curves, and avoidance of delays in the cure of non-COVID-19 patients.

\section{Keypoints}

The Lombardy region in northern Italy was the epicenter of the Coronavirus Disease 2019 (COVID-19) outbreak in the Italian national territory and one of the most afflicted areas worldwide.

The pandemic triggered the urgent need for logistic, organizational, and management adjustments within most hospitals.

Humanitas Clinical and Research Center built a new hospital facility, Emergency Hospital 19 (EH19), during the second wave of the pandemic to increase capacity and optimize infection containment.

The implementation of an additional COVID-19 hospital facility was effective in improving the overall quality of care during the first wave of the pandemic compared with the second wave.

Author Contributions: Conceptualization, A.V., A.D., S.L. and A.G.L.; methodology, E.A., M.K. and G.G.; validation, A.V., S.L. and S.B. (Stefanos Bonovas); formal analysis, D.P., S.B. (Stefanos Bonovas) and G.A.; investigation, V.S., A.A. and M.G. (Mauro Giordano); resources, A.V., E.C., A.L. and C.A.; data curation, A.D., V.S., G.A., A.A. and A.G.L.; writing-original draft preparation, A.V. and S.L.; writing-review and editing, S.L., M.G. (Massimiliano Greco), S.B. (Salvatore Badalamenti) and M.C.; visualization, A.V. and M.C.; supervision, A.V., M.C., V.S., S.B. (Stefanos Bonovas) and S.L.; project administration, A.V. and M.C. All authors have read and agreed to the published version of the manuscript.

Funding: This research received no external funding.

Institutional Review Board Statement: All procedures performed in the study were in accordance with the ethical standards of the institution and with the 1964 Helsinki Declaration and its later amendments or comparable ethical standards.

Informed Consent Statement: Informed consent was obtained from all subjects involved in the study.

Patient and Public Involvement: It was not appropriate or possible to involve patients or the public.

Data Availability Statement: All data are included in the main text.

Conflicts of Interest: All authors report no conflict of interest relevant to this article.

\section{References}

1. Adil, M.T.; Rahman, R.; Whitelaw, D.; Jain, V.; Al-Taan, O.; Rashid, F.; Munasinghe, A.; Jambulingam, P. SARS-CoV-2 and the pandemic of COVID-19. Postgrad. Med. J. 2021, 97, 110-116. [CrossRef] [PubMed]

2. World Health Organization. Clinical Management of Severe Acute Respiratory Infection (SARI) when COVID-19 Disease is Suspected: Interim Guidance, 13 March 2020; World Health Organization: Geneva, Switzerland, 2020.

3. WHO. We Now Have a Name for the \#2019nCoV Disease: COVID-19. Available online: https://twitter.com/WHO/status/1227 248333871173632 (accessed on 1 January 2021).

4. WHO. Clinical Management of COVID-19. Available online: https://www.who.int/publications/i/item/clinical-managementof-covid-19 (accessed on 28 January 2021). 
5. Masetti, C.; Generali, E.; Colapietro, F.; Voza, A.; Cecconi, M.; Messina, A.; Omodei, P.; Angelini, C.; Ciccarelli, M.; Badalamenti, S.; et al. High mortality in COVID-19 patients with mild respiratory disease. Eur. J. Clin. Invest. 2020, 50 , e13314. [CrossRef]

6. Viganò, M.; Voza, A.; Harari, S.; Eusebio, A.; Ripoll Pons, M.; Bordonali, M.; Preti, V.; Rumi, M.G.; Badalamenti, S.; Aghemo, A. Letter to the Editor: Clinical Management of Nonrespiratory Diseases in the COVID-19 Pandemic: What Have We Done and What Needs to Be Done? Telemed. J. E. Health 2020, 26, 1206-1208. [CrossRef]

7. Cecconi, M.; Piovani, D.; Brunetta, E.; Aghemo, A.; Greco, M.; Ciccarelli, M.; Angelini, C.; Voza, A.; Omodei, P.; Vespa, E.; et al. Early Predictors of Clinical Deterioration in a Cohort of 239 Patients Hospitalized for Covid-19 Infection in Lombardy, Italy. J. Clin. Med. 2020, 9, 1548. [CrossRef]

8. Ali, I.; Alharbi, O.M.L. COVID-19: Disease, management, treatment, and social impact. Sci. Total Environ. 2020, $728,138861$. [CrossRef]

9. Shi, Y.; Wang, G.; Cai, X.P.; Deng, J.W.; Zheng, L.; Zhu, H.H.; Zheng, M.; Yang, B.; Chen, Z. An overview of COVID-19. J. Zhejiang Univ. Sci. B 2020, 21, 343-360. [CrossRef]

10. Wang, M.; Liao, Z. SARS-CoV-2 and COVID-19: How much do we know? Acta Virol. 2020, 64, 288-296. [CrossRef] [PubMed]

11. Shamsoddin, E. A COVID-19 pandemic guideline in evidence-based medicine. Evid. Based Dent. 2020, 21, 71-73. [CrossRef] [PubMed]

12. Desai, A.; Caltagirone, G.; Sari, S.; Pocaterra, D.; Kogan, M.; Azzolini, E.; Savevski, V.; Martinelli-Boneschi, F.; Voza, A. The Use of Antiviral Agents against SARS-CoV-2: Ineffective or Time and Age Dependent Result? A Retrospective, Observational Study among COVID-19 Older Adults. J. Clin. Med. 2021, 10, 686. [CrossRef]

13. Desai, A.; Santonocito, O.G.; Caltagirone, G.; Kogan, M.; Ghetti, F.; Donadoni, I.; Porro, F.; Savevski, V.; Poretti, D.; Ciccarelli, M.; et al. Effectiveness of Streptococcus Pneumoniae Urinary Antigen Testing in Decreasing Mortality of COVID-19 Co-Infected Patients: A Clinical Investigation. Med. Kaunas 2020, 56, 572. [CrossRef] [PubMed]

14. Mantica, G.; Riccardi, N.; Terrone, C.; Gratarola, A. Non-COVID-19 visits to emergency departments during the pandemic: The impact of fear. Public Health 2020, 183, 40-41. [CrossRef] [PubMed]

15. Hanft, K. Lean hospitals: Improving quality, patient safety, and employee engagement 2nd edition. HERD Health Environ. Res. Des. J. 2013, 7, 124. [CrossRef] [PubMed]

16. Rotter, T.; Plishka, C.; Lawal, A.; Harrison, L.; Sari, N.; Goodridge, D.; Flynn, R.; Chan, J.; Fiander, M.; Poksinska, B.; et al. What Is Lean Management in Health Care? Development of an Operational Definition for a Cochrane Systematic Review. Eval. Health Prof. 2019, 42, 366-390. [CrossRef]

17. World Medical Association. World Medical Association Declaration of Helsinki: Ethical principles for medical research involving human subjects. JAMA 2013, 310, 2191-2194. [CrossRef]

18. Thomas, L.E.; Li, F.; Pencina, M.J. Overlap Weighting: A Propensity Score Method That Mimics Attributes of a Randomized Clinical Trial. JAMA 2020, 323, 2417-2418. [CrossRef]

19. Li, F.; Thomas, L.E.; Li, F. Addressing Extreme Propensity Scores via the Overlap Weights. Am. J. Epidemiol. 2019, 188, 250-257. [CrossRef]

20. Johns Hopkins Coronavirus Resource Center. Available online: https:/ / coronavirus.jhu.edu/map.html (accessed on 17 February 2021).

21. Percudani, M.; Corradin, M.; Moreno, M.; Indelicato, A.; Vita, A. Mental Health Services in Lombardy during COVID-19 outbreak. Psychiatry Res. 2020, 288, 112980. [CrossRef]

22. Odone, A.; Delmonte, D.; Scognamiglio, T.; Signorelli, C. COVID-19 deaths in Lombardy, Italy: Data in context. Lancet Public Health 2020, 5, e310. [CrossRef]

23. Grasselli, G.; Zangrillo, A.; Zanella, A.; Antonelli, M.; Cabrini, L.; Castelli, A.; Cereda, D.; Coluccello, A.; Foti, G.; Fumagalli, R.; et al. Baseline Characteristics and Outcomes of 1591 Patients Infected With SARS-CoV-2 Admitted to ICUs of the Lombardy Region, Italy. JAMA 2020, 323, 1574-1581. [CrossRef] [PubMed]

24. Wen, C.; Tian, J.D.; Xie, M.; Xu, J.M. Roles of the public-facility-turned temporary hospital in prevention and control of coronavirus disease 2019 in Wuhan, China and clinical experience in the hospital. Zhongguo Dang Dai Er Ke Za Zhi 2020, 22, 409-413. [CrossRef]

25. Luo, H.; Liu, J.; Li, C.; Chen, K.; Zhang, M. Ultra-rapid delivery of specialty field hospitals to combat COVID-19: Lessons learned from the Leishenshan Hospital project in Wuhan. Autom. Constr. 2020, 119, 103345. [CrossRef]

26. Wu, Z.; McGoogan, J.M. Characteristics of and Important Lessons from the Coronavirus Disease 2019 (COVID-19) Outbreak in China: Summary of a Report of 72314 Cases From the Chinese Center for Disease Control and Prevention. JAMA 2020, 323, 1239-1242. [CrossRef]

27. Perlini, S.; Canevari, F.; Cortesi, S.; Sgromo, V.; Brancaglione, A.; Contri, E.; Pettenazza, P.; Salinaro, F.; Speciale, F.; Sechi, G.; et al. Emergency Department and Out-of-Hospital Emergency System (112-AREU 118) integrated response to Coronavirus Disease 2019 in a Northern Italy centre. Intern. Emerg. Med. 2020, 15, 825-833. [CrossRef]

28. Roifman, I.; Arora, R.C.; Bewick, D.; Chow, C.M.; Clarke, B.; Cowan, S.; Ducharme, A.; Gin, K.; Graham, M.; Gupta, A.; et al. Cardiovascular care delivery during the second wave of COVID-19 in Canada. Can. J. Cardiol. 2020, 37, 790-793. [CrossRef]

29. Salenger, R.; Etchill, E.W.; Ad, N.; Matthew, T.; Alejo, D.; Whitman, G.; Lawton, J.S.; Lau, C.L.; Gammie, C.F.; Gammie, J.S. The Surge After the Surge: Cardiac Surgery Post-COVID-19. Ann. Thorac. Surg. 2020, 110, 2020-2025. [CrossRef] 
30. Tartaglia, N.; Pavone, G.; Lizzi, V.; Vovola, F.; Tricarico, F.; Pacilli, M.; Ambrosi, A. How emergency surgery has changed during the COVID-19 pandemic: A cohort study. Ann. Med. Surg. Lond. 2020, 60, 686-689. [CrossRef]

31. Serrano, O.K.; Orlando, R.; Papasavas, P.; McClure, M.H.; Kumar, A.; Steinberg, A.C.; Cohen, J.L.; Shichman, S.J.; Singh, R.K.; Sardella, W.V.; et al. Getting back to work: A framework and pivot plan to resume elective surgery and procedures after COVID-19. Surg. Open. Sci. 2021, 4, 12-18. [CrossRef]

32. Pelargos, P.E.; Chakraborty, A.R.; Adogwa, O.; Swartz, K.; Zhao, Y.D.; Smith, Z.A.; Dunn, I.F.; Bauer, A.M. An Evaluation of Neurosurgical Practices During the Coronavirus Disease 2019 Pandemic. World Neurosurg. 2021, 146, e91-e99. [CrossRef] [PubMed]

33. Campi, R.; Amparore, D.; Capitanio, U.; Checcucci, E.; Salonia, A.; Fiori, C.; Minervini, A.; Briganti, A.; Carini, M.; Montorsi, F.; et al. Assessing the Burden of Nondeferrable Major Uro-oncologic Surgery to Guide Prioritisation Strategies During the COVID-19 Pandemic: Insights from Three Italian High-volume Referral Centres. Eur. Urol. 2020, 78, 11-15. [CrossRef] [PubMed]

34. Lee, A.K.F.; Cho, R.H.W.; Lau, E.H.L.; Cheng, H.K.; Wong, E.W.Y.; Ku, P.K.M.; Chan, J.Y.K.; Yeung, Z.W.C. Mitigation of head and neck cancer service disruption during COVID-19 in Hong Kong through telehealth and multi-institutional collaboration. Head Neck 2020, 42, 1454-1459. [CrossRef] [PubMed]

35. Ferrara, P.; Albano, L. COVID-19 and healthcare systems: What should we do next? Public Health 2020, 185, 1-2. [CrossRef] [PubMed]

36. Breen, L.M.; Trepp, R., Jr.; Gavin, N. Lean Process Improvement in the Emergency Department. Emerg. Med. Clin. N. Am. 2020, 38, 633-646. [CrossRef]

37. Aij, K.H.; Teunissen, M. Lean leadership attributes: A systematic review of the literature. J. Health Organ. Manag. 2017, 31, 713-729. [CrossRef]

38. White, B.A.; Baron, J.M.; Dighe, A.S.; Camargo, C.A., Jr.; Brown, D.F. Applying Lean methodologies reduces ED laboratory turnaround times. Am. J. Emerg. Med. 2015, 33, 1572-1576. [CrossRef] [PubMed]

39. Holden, R.J. Lean Thinking in emergency departments: A critical review. Ann. Emerg. Med. 2011, 57, 265-278. [CrossRef] [PubMed]

40. Shah, R.; Ward, P. Defining and Developing Measures of Lean Production. J. Oper. Manag. 2007, 25, 785-805. [CrossRef] 